\title{
Impact of monocyte to high-density lipoprotein ratio on prevalent hyperuricemia: findings from a rural Chinese population
}

\author{
Meng-Qi Chen ${ }^{1+}$, Wen-Rui Shi ${ }^{1 \dagger}$, Chu-Ning Shi ${ }^{1}$, Ya-Ping Zhou ${ }^{2}$ and Ying-Xian Sun ${ }^{1 *}$
}

\begin{abstract}
Background: Monocyte to high-density lipoprotein cholesterol ratio (MHR) is a novel inflammatory marker that has been used to predict various inflammation-related diseases. This study aims to explore the association between MHR and prevalent hyperuricemia in a rural Chinese population.

Methods: 8163 eligible participants (mean age: 54.13 years, males: 45.71\%) from northeast China were enrolled in this cross-sectional study between 2012 to 2013. MHR was determined as blood monocyte count ratio to highdensity lipoprotein cholesterol concentration.

Results: The prevalence of hyperuricemia was $12.86 \%$. After adjusting for potential confounding factors, per SD increase of MHR caused a $25.2 \%$ additional risk for hyperuricemia, and the top quartile of MHR had an $82.9 \%$ increased risk for hyperuricemia compared with the bottom quartile. Additionally, smooth curve fitting and subgroup analyses showed a linear and robust association between MHR and prevalent hyperuricemia respectively. Finally, after introducing MHR into the established model of risk factors, the AUC displayed a significant improvement $(0.718$ vs $0.724, p=0.008)$. Furthermore, Category-free net reclassification improvement $(0.160,95 \% \mathrm{Cl}: 0.096-0.224, P<0.001)$ and integrated discrimination improvement $(0.003,95 \% \mathrm{Cl}: 0.002-0.005, P<0.001)$ also demonstrated significant improvements.

Conclusions: The present study suggests that MHR was positively and independently correlated with prevalent hyperuricemia among rural Chinese adults. Our results also implicate an important value for MHR in optimizing the risk stratification of hyperuricemia.
\end{abstract}

Keywords: Hyperuricemia, Monocyte to high-density lipoprotein ratio, Inflammation, Chinese population

\footnotetext{
* Correspondence: sunyingxian_1962@163.com

${ }^{+}$Meng-Qi Chen and Wen-Rui Shi contributed equally to this work.

'Department of Cardiology, The First Hospital of China Medical University,

155 Nanjing North Street, Heping District, Shenyang 110001, China

Full list of author information is available at the end of the article
}

(c) The Author(s). 2020 Open Access This article is licensed under a Creative Commons Attribution 4.0 International License, which permits use, sharing, adaptation, distribution and reproduction in any medium or format, as long as you give appropriate credit to the original author(s) and the source, provide a link to the Creative Commons licence, and indicate if changes were made. The images or other third party material in this article are included in the article's Creative Commons licence, unless indicated otherwise in a credit line to the material. If material is not included in the article's Creative Commons licence and your intended use is not permitted by statutory regulation or exceeds the permitted use, you will need to obtain permission directly from the copyright holder. To view a copy of this licence, visit http://creativecommons.org/licenses/by/4.0/ The Creative Commons Public Domain Dedication waiver (http://creativecommons.org/publicdomain/zero/1.0/) applies to the data made available in this article, unless otherwise stated in a credit line to the data. 


\section{Background}

In recent decades, hyperuricemia is becoming a major public health issue due to its close association with critical diseases such as gout, hypertension, diabetes mellitus, chronic kidney disease and other cardiovascular diseases (CVD) [16]. For example, the Mendelian randomization study supported that hyperuricemia may play a causal role in the development of CVD, suggesting its great significance for early screening and prevention of CVD [5]. Accordingly, there is a clear need for a simple approach to improve the risk stratification and prevention of hyperuricemia.

Chronic inflammation is a pathophysiological process characterized by elevated inflammatory mediators which closely associate with hyperuricemia [7, 8]. Epidemiological reports have demonstrated a significant correlation between inflammation and elevated uric acid level $[2,9]$. Consistently, previous studies have shown that hyperuricemia might induce inflammation by activating the expression of inflammatory mediators [10, 11]. Therefore, these studies indicated that hyperuricemia was closely related to inflammation.

Monocytes and high-density lipoprotein cholesterol (HDL-C) are two important factors in the development of inflammation [12-14]. Monocytes interact mainly with platelets and endothelial cells, leading to aggravation in inflammation and prothrombotic pathways [15, 16]. In contrast, HDL-C protects endothelial cells from oxidative stress and inflammation by regulating monocytes activation and monocyte progenitors proliferation, and preventing monocytes assembly into the arterial wall [12-14]. High monocyte counts and low HDL-C levels have been shown to be positively associated with inflammation [17-19]. Therefore, while monocytes play a pro-inflammatory role, HDL-C functions as a reverse factor in the inflammatory process.

Monocyte-to-HDL-C ratio (MHR) has been proposed as a potentially modifiable marker of inflammation [20]. Additionally, previous studies have found that MHR has the capacity to predict a variety of inflammation-related diseases [21-24]. As yet, no study has been conducted on the potential association between MHR and the prevalent hyperuricemia. Therefore, our study used data from the Northeast China Rural Cardiovascular Health Study (NCRCHS) to investigate this relationship and explore the value of MHR to optimize the risk stratification of hyperuricemia.

\section{Methods}

\section{Study population}

The present study was based on a large scale crosssectional epidemiological survey known as NCRCHS that conducted from January 2012 to August 2013. The detailed design and rationale of NCRCHS were fully described elsewhere [25]. 14,016 permanent residents (age $\geq 35$ years) from rural areas of Northeast China were recruited to assess the incidence, prevalence, and natural history of cardiovascular risk factors. Participants were selected by the scheme of a multistage and stratified random sampling. First, three counties of Dawa, Zhangwu, and Liaoyang were selected from the eastern, southern, and northern regions of Liaoning province. Afterward, three towns were randomly selected from three counties. In the end, a total of 26 rural villages were randomly selected. Due to 2060 subjects failed to complete the study, 11,956 individuals were included in our study, producing a response rate of $85.3 \%$. Moreover, 3793 subjects were further excluded for missing biochemical and clinical data. Finally, we enrolled 8163 eligible participants into the present analysis (Fig. 1). Our study was approved by the Ethics Committee of China Medical University (Shenyang, China). Written informed consent was voluntarily signed by all participants; if disabled, informed consent was obtained from the proxies of the subjects.

\section{Data collection and measurements}

Previously published studies have fully reported the methods of data collection and measurement [26, 27]. Before the study, cardiologists and nurses must pass a strict exam to obtain the qualification for conducting questionnaires which collect baseline information about sociodemographic data, health-related behaviors, anthropometric indexes, and history of CVD. Quality assurance of the data collection process was executed by the central steering committee with a subcommittee. The race of participants was separated into Han and others. Education level was split into three categories: primary school or below, middle school, and high school or above. Family annual income of the subjects was classified into three groups: $\leq 5000,5000-20,000$ and $>20,000 \mathrm{CNY}$ per year. Physical activity was categorized into three levels of low, middle and high based on the self-reports of individuals. Use of lipid-lowering drug was determined as lipid drug uptake over the past 2 weeks. History of CVD included angina pectoris, myocardial infarction, atrial fibrillation, arrhythmia, and heart failure.

The blood pressure of participants was measured three times after at least $5 \mathrm{~min}$ of rest in a completely relaxed and sitting position. The result of blood pressure for analysis was the average of three consecutive readings taken by two randomly selected staff.

Concerning anthropometric measurements, individuals were required to wear light clothes without shoes. Calibrated digital scales were applied to measure the standard weight to the nearest $0.1 \mathrm{~kg}$. To quantify standard height with a calibrated stadiometer, subjects were asked to remain standing position. And we recorded the reading of height to the nearest $0.1 \mathrm{~cm}$. Anthropometric 


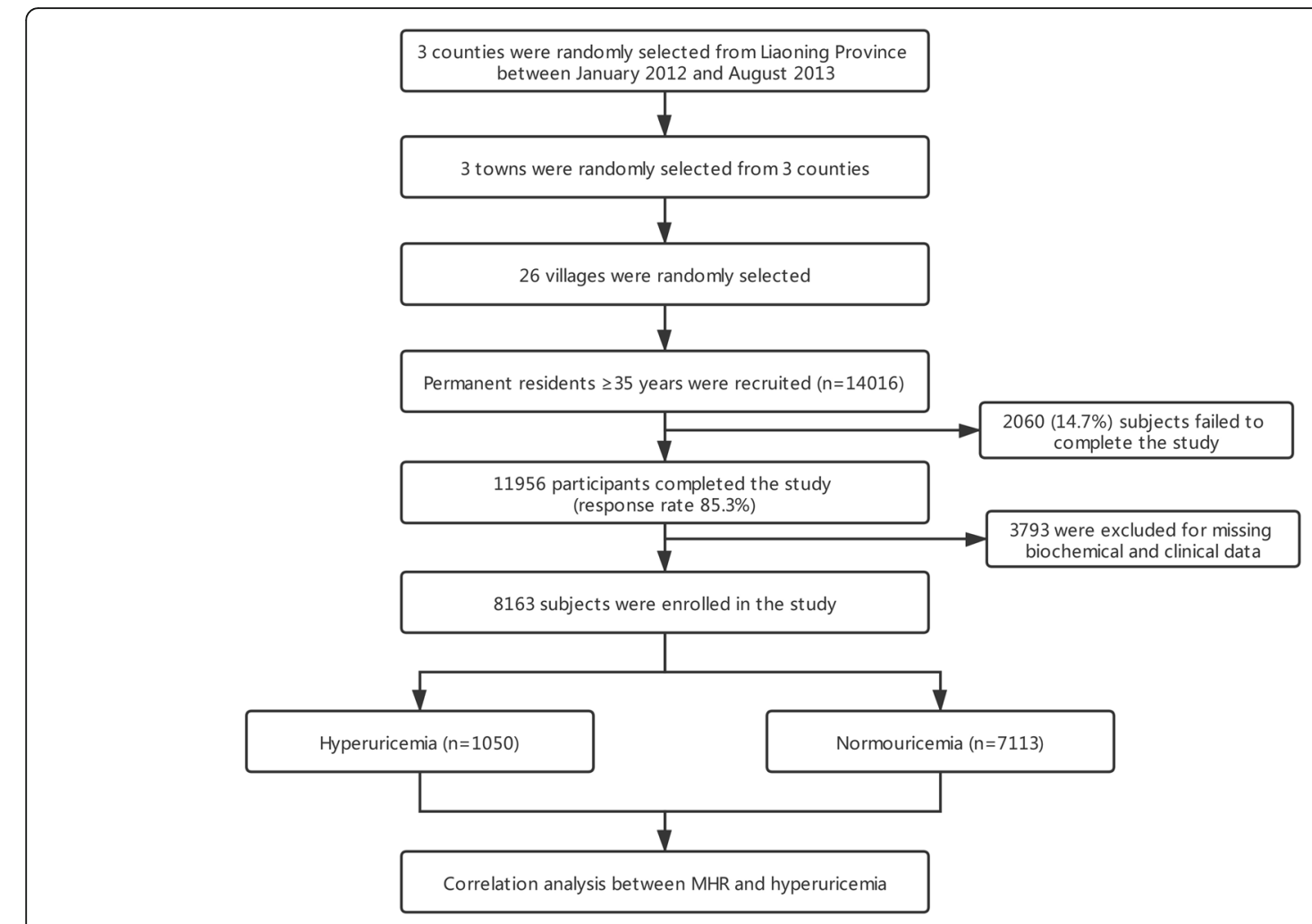

Fig. 1 Flowchart describing the recruitment process and generation of study population

measurements were taken twice and the mean values were used into the analysis.

Fasting blood samples of the antecubital veins were gathered in the morning after all participants had fasted for at least $12 \mathrm{~h}$. Venous blood samples were separated into serum samples through a standard centrifuge, and they were transported by EDTA tubes. Finally, the samples were frozen at $-20 \mathrm{C}$ degree for a better analysis of serum creatinine (Scr), fasting plasma glucose (FPG), triglyceride (TG), total cholesterol (TC), low-density lipoprotein cholesterol (LDL-C), HDL-C, white blood cells (WBC), lymphocytes, neutrophils and monocytes.

\section{Definition}

Body mass index (BMI) was calculated as mean weight divided by mean height squared $(\mathrm{kg} / \mathrm{m} 2)$. The estimated glomerular filtration rate (eGFR) was defined according to the CKD-EPI (Chronic Kidney Disease Epidemiology Collaboration) equation [28]. MHR was determined as blood monocyte count ratio to high-density lipoprotein cholesterol concentration [29]. The definition of hyperuricemia was serum uric acid (SUA) $\geq 357 \mu \mathrm{mol} / \mathrm{L}(6 \mathrm{mg} / \mathrm{dL})$ for females and $\geq 417 \mu \mathrm{mol} / \mathrm{L}(7 \mathrm{mg} / \mathrm{dL})$ for males [30].

\section{Statistical analysis}

Continuous variables were presented as mean values \pm standard deviation (SD) or median (interquartile range) based on the distribution. Category variables were displayed as frequencies (percentages). Students' t-test or Mann-Whitney test was applied to compare continuous variables between groups. Chi-square test was adopted to compare categorical variables between groups. In addition, the rank-sum test was employed to make the utmost of ordinal information for ordinal category variables. Multivariate logistic regression was utilized to demonstrate the independent relationship between MHR and the prevalence of hyperuricemia. Odds ratio (OR) and 95\% confidence interval $(95 \% \mathrm{CI})$ were presented in the results. The linear relationship between normalized MHR and the prevalence of hyperuricemia was explored by a spline smoothing function with a generalized additive model. Subgroup analyses were tested to detect the robustness of the association between MHR and the prevalent hyperuricemia. Finally, receiver operating characteristic (ROC) curve, integrated discrimination improvement (IDI) and category-free net reclassification improvement (NRI) was employed to estimate the potential of MHR to enhance the risk classification of hyperuricemia. The whole statistical analyses were performed by SPSS 25.0 software (IBP corp), EmpowerStats (http://www.empowerstats.com, X\&Y Solutions, Inc., Boston, MA) and statistical software packages $\mathrm{R}$ (http://www.R-project.org, The R Foundation). Statistical significance was identified by a two-tailed $P$ value $<0.05$. 


\section{Results}

Table 1 summarizes the baseline characteristics of 8163 subjects (45.71\% men and $54.29 \%$ women). The crude prevalence of hyperuricemia was $12.86 \%$. As for demographic data, population with hyperuricemia were older and had a higher proportion of male as well as the Han

Table 1 Characteristics of subjects stratified by hyperuricemia

\begin{tabular}{|c|c|c|c|c|}
\hline Variables & Total $(n=8163)$ & Hyperuricemia $(n=1050)$ & Normouricemia $(n=7113)$ & $P$ value $^{a}$ \\
\hline Age (years) & $54.13 \pm 10.49$ & $55.12 \pm 10.96$ & $53.99 \pm 10.41$ & 0.001 \\
\hline Males (\%) & $3731(45.71)$ & $652(62.10)$ & $3079(43.29)$ & $<0.001$ \\
\hline Race, Han (\%) & $7936(97.22)$ & $1033(98.38)$ & $6903(97.05)$ & 0.014 \\
\hline Education (\%) & & & & 0.716 \\
\hline Primary school or below & $4322(52.95)$ & $561(53.43)$ & $3761(52.88)$ & \\
\hline Middle school & $3134(38.39)$ & $393(37.43)$ & $2741(38.54)$ & \\
\hline High school or above & 707 (8.66) & $96(9.14)$ & $611(8.59)$ & \\
\hline Income, CNY (\%) & & & & 0.012 \\
\hline$\leq 5000$ & $851(10.43)$ & $134(12.76)$ & $717(10.08)$ & \\
\hline $5000-20,000$ & $4294(52.60)$ & $557(53.05)$ & $3737(52.54)$ & \\
\hline$>20,000$ & $3018(36.97)$ & $359(34.19)$ & $2659(37.38)$ & \\
\hline Physical activity (\%) & & & & 0.101 \\
\hline Low & $3192(39.10)$ & $436(41.52)$ & $2756(38.75)$ & \\
\hline Middle & $1547(18.95)$ & $205(19.52)$ & $1342(18.87)$ & \\
\hline High & $3424(41.95)$ & 409 (38.95) & $3015(42.39)$ & \\
\hline Current smoking (\%) & $2767(33.90)$ & $398(37.90)$ & $2369(33.31)$ & 0.003 \\
\hline Current drinking (\%) & $1734(21.24)$ & 332 (31.62) & $1402(19.71)$ & $<0.001$ \\
\hline Height (cm) & $160.66 \pm 8.15$ & $162.97 \pm 8.45$ & $160.32 \pm 8.05$ & $<0.001$ \\
\hline Weight (kg) & $63.49 \pm 11.27$ & $69.85 \pm 12.16$ & $62.56 \pm 10.82$ & $<0.001$ \\
\hline $\mathrm{BMI}\left(\mathrm{kg} / \mathrm{m}^{2}\right)$ & $24.54 \pm 3.61$ & $26.26 \pm 3.85$ & $24.29 \pm 3.50$ & $<0.001$ \\
\hline $\mathrm{SBP}(\mathrm{mmHg})$ & $138.68 \pm 21.93$ & $144.79 \pm 23.44$ & $137.77 \pm 21.55$ & $<0.001$ \\
\hline $\mathrm{DBP}(\mathrm{mmHg})$ & $81.86 \pm 11.64$ & $86.09 \pm 12.63$ & $81.24 \pm 11.35$ & $<0.001$ \\
\hline $\operatorname{Scr}(\mu \mathrm{mol} / \mathrm{L})$ & $73.80(66.70-82.20)$ & $82.70(74.73-91.80)$ & $72.80(66.10-80.70)$ & $<0.001$ \\
\hline eGFR $\left(\mathrm{ml} / \mathrm{min}\right.$ per $\left.1.73 \mathrm{~m}^{2}\right)$ & $89.33 \pm 15.42$ & $81.92 \pm 19.13$ & $90.42 \pm 14.48$ & $<0.001$ \\
\hline $\mathrm{FPG}(\mathrm{mmol} / \mathrm{L})$ & $5.60(5.22-6.10)$ & $5.72(5.32-6.35)$ & $5.58(5.22-6.06)$ & $<0.001$ \\
\hline $\mathrm{TC}(\mathrm{mmol} / \mathrm{L})$ & $5.32 \pm 1.11$ & $5.63 \pm 1.28$ & $5.27 \pm 1.07$ & $<0.001$ \\
\hline $\mathrm{TG}(\mathrm{mmol} / \mathrm{L})$ & $1.29(0.91-1.96)$ & $1.88(1.28-2.87)$ & $1.23(0.88-1.83)$ & $<0.001$ \\
\hline $\mathrm{HDL}-\mathrm{C}(\mathrm{mmol} / \mathrm{L})$ & $1.34 \pm 0.32$ & $1.28 \pm 0.33$ & $1.35 \pm 0.32$ & $<0.001$ \\
\hline $\mathrm{LDL}-\mathrm{C}(\mathrm{mmol} / \mathrm{L})$ & $2.89 \pm 0.80$ & $3.07 \pm 0.88$ & $2.87 \pm 0.79$ & $<0.001$ \\
\hline WBC count $\left(10^{9} / \mathrm{L}\right)$ & $6.20 \pm 2.06$ & $6.61 \pm 1.79$ & $6.14 \pm 2.09$ & $<0.001$ \\
\hline Lymphocyte count $\left(10^{9} / \mathrm{L}\right)$ & $1.90(1.60-2.40)$ & $2.10(1.70-2.50)$ & $1.90(1.60-2.30)$ & 0.017 \\
\hline Neutrophil count $\left(10^{9} / \mathrm{L}\right)$ & $3.40(2.70-4.30)$ & $3.70(2.90-4.60)$ & $3.40(2.60-4.20)$ & $<0.001$ \\
\hline Monocyte count $\left(10^{9} / \mathrm{L}\right)$ & $0.41(0.30-0.60)$ & $0.50(0.40-0.70)$ & $0.40(0.30-0.60)$ & $<0.001$ \\
\hline Lipid-lowering drug (\%) & $272(3.33)$ & $78(7.43)$ & $194(2.73)$ & $<0.001$ \\
\hline History of CVD (\%) & $1268(15.53)$ & $222(21.14)$ & $1046(14.71)$ & $<0.001$ \\
\hline SUA $(\mu \mathrm{mol} / \mathrm{L})$ & $300.77 \pm 85.68$ & $452.12 \pm 70.17$ & $278.43 \pm 61.79$ & $<0.001$ \\
\hline MHR & $0.38 \pm 0.25$ & $0.45 \pm 0.27$ & $0.37 \pm 0.24$ & $<0.001$ \\
\hline
\end{tabular}

Data are expressed as mean \pm standard deviation (SD) or median (interquartile range) and numbers (percentage) as appropriate

Abbreviations: $C N Y$ Chinese currency (1CNY $=0.15$ USD), BMI body mass index, SBP systolic blood pressure, DBP diastolic blood pressure, SCr serum creatinine, eGFR estimated glomerular filtration rate, FPG fasting plasma glucose, $T C$ total cholesterol, $T G$ triglyceride, $H D L-C$ high-density lipoprotein cholesterol, $L D L-C$ low-density lipoprotein cholesterol, WBC white blood cell, CVD cardiovascular disease, SUA serum uric acid, MHR Monocyte to high-density lipoprotein ratio

${ }^{a}$ Comparisons for category variables between groups were tested by $\mathrm{X} 2$ test or rank-sum test (ordinal category variables) and comparisons of continuous variables between groups were tested by Student's t-test or Mann-Whitney test 
race than the healthy group. Furthermore, hyperuricemia participants had lower family annual income and more likely to be a current drinker or smoker compared with normouricemia subjects. About the anthropometric characteristics, hyperuricemia group had significantly higher levels of height, weight, BMI, SBP, and DBP. Laboratory examinations exhibited higher Scr, FPG, TC, TG, and LDL-C concentrations together with greater numbers of white blood cells, lymphocytes, neutrophils and monocytes in the patients' group. Additionally, hyperuricemia patients had markedly lower eGFR and HDL-C levels. Moreover, the percentages of CVD history, and lipidslowering drug were statistically augmented in the hyperuricemia subjects. Lastly, we could observe a substantially greater level of MHR in the hyperuricemia group than the normouricemia group (all $P<0.05$ ).

Logistic regression analyses revealed the association between MHR and prevalent hyperuricemia, as displayed in Table 2. In model 2, per SD increase of MHR caused a $37.5 \%$ additional risk for hyperuricemia after adjusting for age, sex, race, education level, family annual income and physical activity, current smoking and drinking status. After additional adjustment of BMI, eGFR, TC, HDL-C, SBP, FPG, lipidlowering drug, and CVD history, the risk attenuated to $25.2 \%$. When dividing MHR into quartiles, we could observe the top quartile had an $82.9 \%$ increased risk for hyperuricemia compared with the bottom quartile in the fully adjusted model. Furthermore, the prevalence of hyperuricemia displayed a significant linear trend across the quartiles ( $\mathrm{P}$ for trend $<0.001$ ).

To further demonstrate the linear association between MHR and the prevalence of hyperuricemia, we performed a smooth curve fitting with full adjustment of all covariates (Fig. 2). The curve showed a linear correlation between normalized MHR and the risk of hyperuricemia, and the result confirmed the linear trend in the abovedescribed quartile analysis of logistic regression.
To investigate whether the relationship between MHR and hyperuricemia was robust in the logistic regression model, stratified analyses were conducted using several identified risk factors (including age, sex, BMI, SBP, FPG, and eGFR) for hyperuricemia (Fig. 3). After adjusting for the above-described covariates except for the covariate used for stratification, the results of the subgroup analyses revealed the robust association between MHR and hyperuricemia (all $\mathrm{P}$ for interaction $>0.05$ ).

Finally, through ROC analysis and reclassification analyses, the results illuminated the ability of MHR to optimize the risk stratification of hyperuricemia (Table 3). The AUC of MHR for hyperuricemia was 0.598 (95\% CI: $0.587-0.608, P<0.001$ ), and the AUC of the model of several clinical risk factors (including age, sex, current smoking, current drinking, BMI, eGFR, TC, HDL-C, SBP, FPG and history of CVD) was 0.718 (95\% CI: 0.709-0.728, $P<0.001)$. When adding MHR into clinical risk factors, we could detect a statistical increment of AUC (0.724, 95\% CI: 0.715-0.734, P<0.001). Additionally, both the category free NRI $(0.160,95 \% \mathrm{CI}$ : $0.096-0.224, P<0.001)$ and IDI $(0.003,95 \%$ CI: $0.002-$ $0.005, P<0.001)$ showed a significant improvement when adding MHR into above risk factors.

\section{Discussion}

Our study for the first time implicated the impact of MHR on the prevalent hyperuricemia in the general population. Furthermore, the findings revealed the association between MHR and the prevalence of hyperuricemia was linear and robust. Additionally, our analyses suggest that MHR may have the capacity to optimize the risk stratification of hyperuricemia. Taken together, our findings suggest the strong association between MHR and the prevalent hyperuricemia, and the value of MHR to optimize the risk stratification of hyperuricemia.

Table 2 Multivariate logistic regression of MHR for hyperuricemia

\begin{tabular}{|c|c|c|c|c|c|c|}
\hline \multirow[t]{2}{*}{ Variables } & \multicolumn{6}{|c|}{ Odds Ratio $(95 \% \mathrm{Cl})$} \\
\hline & Model 1 & $P$ value & Model 2 & $P$ value & Model 3 & $P$ value \\
\hline MHR (per SD change) & $1.394(1.299,1.496)$ & $<0.001$ & $1.375(1.280,1.478)$ & $<0.001$ & $1.252(1.157,1.355)$ & $<0.001$ \\
\hline \multicolumn{7}{|l|}{ Quartiles of MHR } \\
\hline Quartile 1 & Reference & & Reference & & Reference & \\
\hline Quartile 2 & $1.272(1.035,1.564)$ & 0.022 & $1.243(1.009,1.531)$ & 0.041 & $1.194(0.963,1.480)$ & 0.106 \\
\hline Quartile 3 & $1.447(1.183,1.771)$ & $<0.001$ & $1.392(1.135,1.708)$ & 0.002 & $1.264(1.019,1.569)$ & 0.033 \\
\hline Quartile 4 & $2.418(2.001,2.920)$ & $<0.001$ & $2.320(1.913,2.813)$ & $<0.001$ & $1.829(1.477,2.264)$ & $<0.001$ \\
\hline$P$ for trend & & $<0.001$ & & $<0.001$ & & $<0.001$ \\
\hline
\end{tabular}

Model 1: no adjustment; Model 2: adjusted for age, sex, race, education level, family annual income, physical activity, current smoking, current drinking; Model 3: adjusted for all the factors in model 2 and BMI, eGFR, TC, HDL-C, SBP, FPG, lipid-lowering drug, history of CVD

Abbreviations: MHR Monocyte to high-density lipoprotein ratio, $O R$ odds ratio, $\mathrm{Cl}$ confidence interval, $S D$ standard deviation. Other abbreviations as in Table 1 Quartile 1: MHR $<0.227$; Quartile 2: $0.227 \leq$ MHR $<0.339$; Quartile 3: $0.339 \leq$ MHR $<0.484$; Quartile 4: MHR $\geq 0.484$ 


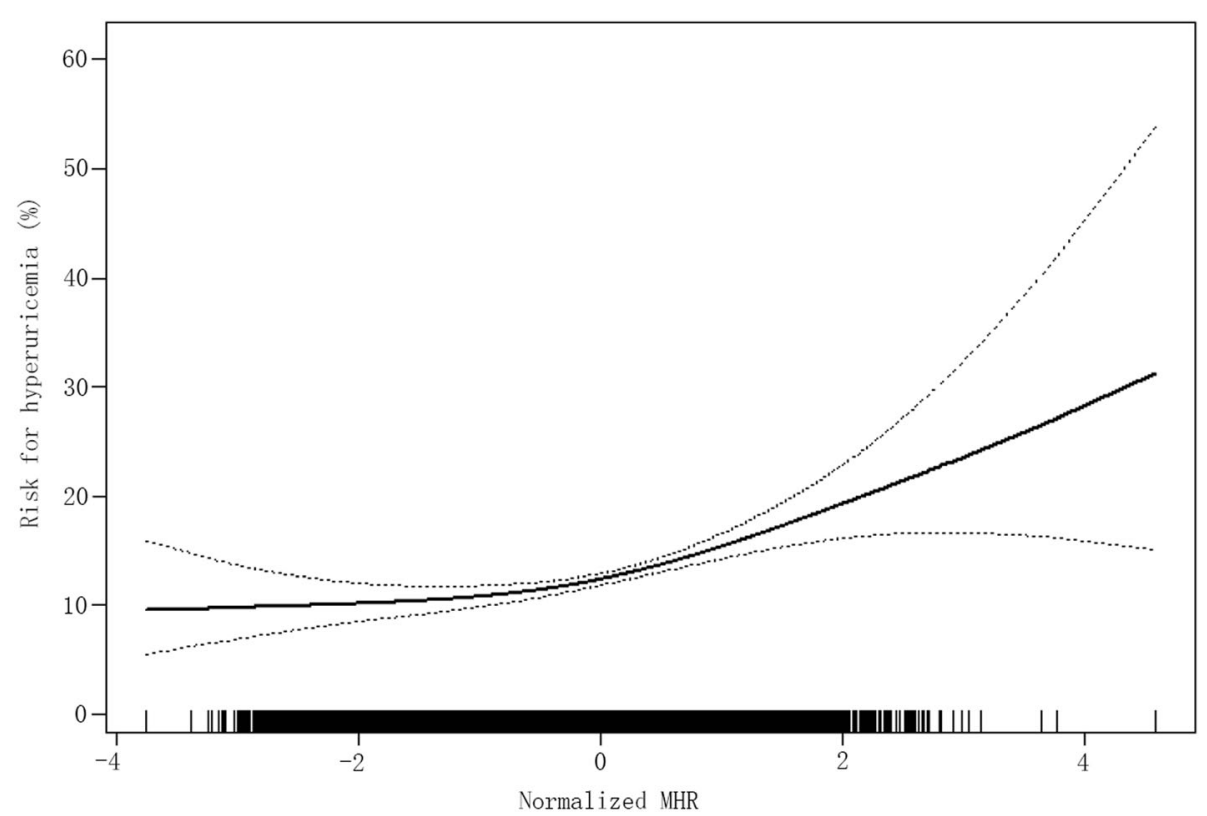

Fig. 2 Smooth curve fitting was employed using generalized additive model to investigate the association between MHR and the risk of hyperuricemia after adjusting for age, sex, race, education level, family annual income, physical activity, current smoking, current drinking, BMI, eGFR, TC, HDL-C, SBP, FPG, lipid-lowering drug, history of CVD. In the figure, the solid line represents the estimated risk of hyperuricemia, and the dotted line indicates pointwise $95 \%$ confidence interval

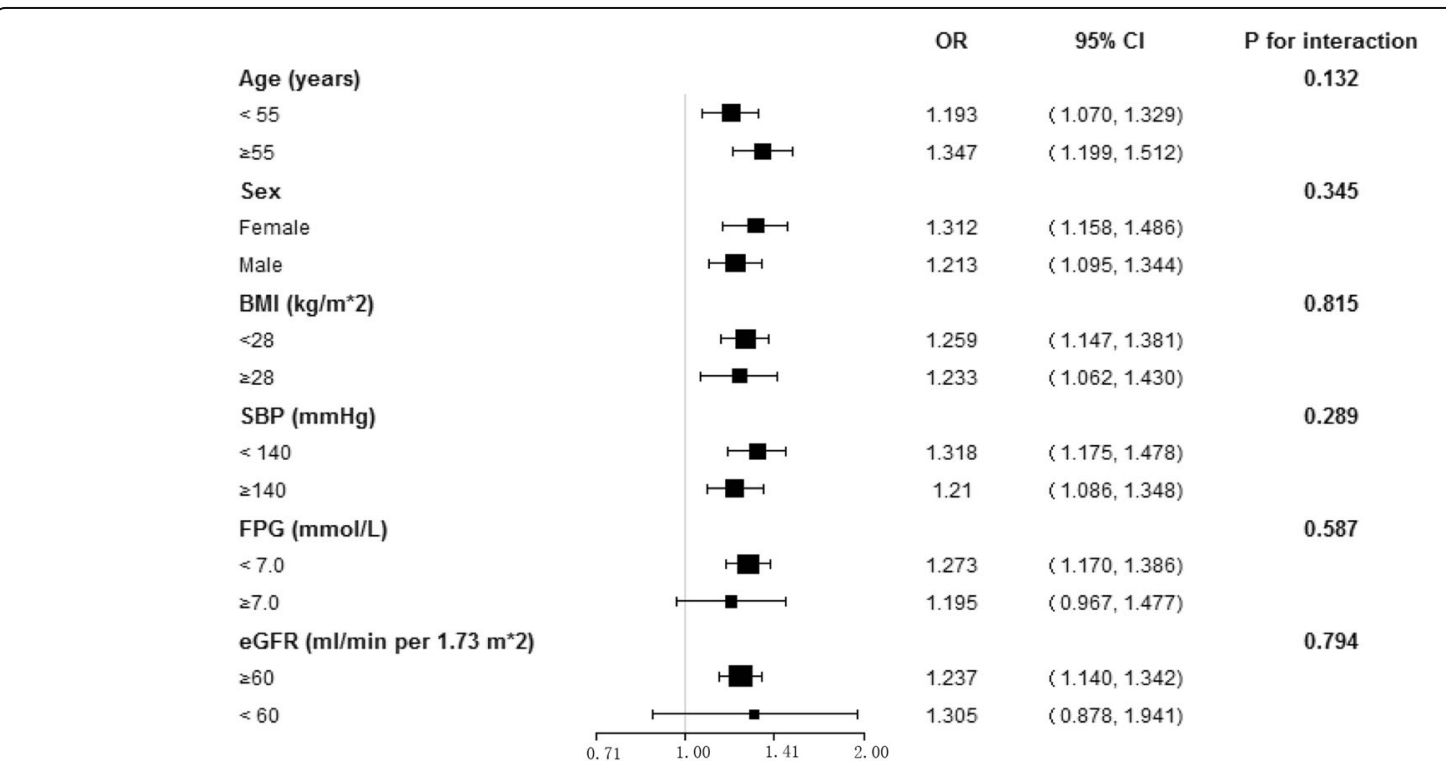

Fig. 3 Subgroup analysis for the impact of MHR on the prevalence of hyperuricemia. The dots and lines represent the estimates of the odds ratios of hyperuricemia for per SD increment of MHR and the corresponding 95\% confidence intervals, respectively. The model adjusted for sex, age, race, education level, family annual income, physical activity, current smoking, current drinking, BMI, eGFR, TC, HDL-C, SBP, FPG, lipid-lowering drug, history of CVD, except for the variable that is stratified 
Table 3 Receiver operating characteristic and reclassification analyses for MHR to discriminate the risk stratification of hyperuricemia

\begin{tabular}{llllllll}
\hline Model & AUC $(95 \% \mathrm{Cl})$ & $P$ value & $P$ for comparison & NRI (category free) & $P$ value & IDI & \\
\hline MHR & $0.598(0.587,0.608)$ & $<0.001$ & - & - & - & - & - \\
Clinical risk factors* & $0.718(0.709,0.728)$ & $<0.001$ & Reference & Reference & Reference & Reference & Reference \\
Clinical risk factors + MHR & $0.724(0.715,0.734)$ & $<0.001$ & 0.008 & $0.160(0.096,0.224)$ & $<0.001$ & $0.003(0.002,0.005)$ & $<0.001$
\end{tabular}

* Clinical risk factors: age, sex, current smoking, current drinking, BMI, eGFR, TC, HDL-C, SBP, FPG, history of CVD

Abbreviations: $M H R$ Monocyte to high-density lipoprotein ratio, $A U C$ area under the curve, $C I$ confidence interval, NRI net reclassification improvement, IDI integrated discrimination improvement, $B M I$ body mass index, eGFR estimated glomerular filtration rate, $T C$ total cholesterol, $H D L-C$ high density lipoprotein cholesterol, SBP systolic blood pressure, FPG fasting plasma glucose, CVD cardiovascular disease

Monocyte counts and HDL-C levels are two pivotal hallmarks in the development and progression of inflammation [12-14]. Chronic inflammation is a systematic process accompanied by an elevation of inflammatory mediators such as acute-phase proteins, cytokines, and adhesion molecules [7]. Monocytes are crucial immune system cells that play a unique role in the inflammatory response [13, 31]. Activated monocyte interacts with activated or damaged endothelial cells, leading to overexpression of proinflammatory cytokines and adhesion molecules, including vascular cell adhesion molecule 1, monocyte chemotactic protein 1 ligand and intercellular adhesion molecule 1 . Thereafter, monocytes move to the subendothelial space and then differentiate into macrophages that engulf oxidized low-density lipoprotein cholesterol [32]. These cells then transform into foam cells that release pro-inflammatory cytokines, attracting more monocytes into the site of inflammation [13, 33]. However, HDL-C plays a key role in the anti-inflammatory effect. HDL-C counteracts the proinflammatory response of monocytes by interrupting the differentiation of monocyte to macrophage and preventing the recruitment of monocyte into vascular wall, as well as prohibiting LDL-C oxidation in the arterial wall [14, 34]. Additionally, HDL-C inhibits the proliferation of monocyte progenitor cells [35]. Therefore, monocytes exhibit proinflammatory effects, but HDL-C acts as a reverse factor in the process of inflammation.

MHR as the combination of both monocytes and HDL-C has been proposed as a novel inflammatory index [20]. Recent clinical and epidemiological studies have established that MHR has the ability to predict multiple inflammation-related diseases, such as metabolic syndrome, diabetes mellitus, atrial fibrillation and coronary artery disease [21-24]. Recent researches have revealed the significant association between inflammation and elevated uric acid level $[2,9]$. For example, Creactive protein (CRP), a marker of low-grade inflammation, was positively correlated with SUA levels [36, 37]. Furthermore, uric acid can induce CRP mRNA expression in vascular endothelium and smooth muscle cells [38]. Hence, these studies indicated a close relationship between SUA and inflammation. In view of the foregoing, we hypothesize that MHR, a novel inflammatory index, also has a significant association with hyperuricemia and the ability to improve risk stratification of hyperuricemia.

The current findings were consistent with our hypothesis. Regardless of whether MHR was used as a continuous variable or a category variable, the results of the logistic regression analysis showed a significant positive correlation between MHR and hyperuricemia. Moreover, we further testified the linear association of MHR with hyperuricemia via performing smooth curve fitting analysis. Therefore, higher MHR suggests a proportionally higher prevalence of hyperuricemia, excluding the presence of threshold or saturation effects. Additionally, in the subgroup analyses of age, sex, BMI, SBP, FPG and eGFR, the stable relationship between MHR and hyperuricemia suggests that the risk stratification capacity of MHR is applicable to these designated populations.

Recently, a substantial proportion of studies reported that serum uric acid was independently associated with chronic kidney diseases [39-42], and it has been demonstrated that MHR was independently associated with reduced renal function [43]. However, our study found that there was not a statistically significant correlation between the MHR and the prevalent hyperuricemia in individuals with an eGFR of less than $60 \mathrm{ml} / \mathrm{min}$ per $1.73 \mathrm{~m}^{2}$. The main reason may be the decrease of statistic power because of the small sample size of participants with hyperuricemia and reduced renal function simultaneously. Similar to our finding, one Mendelian randomization study, using uric acid transporter genetic risk score to explore the causality between serum uric acid and kidney function, suggested that a possible causal relationship between serum uric acid levels and improved renal function in healthy men rather than and reduced renal function [44]. In any case, further study is needed to confirm the current results.

ROC and reclassification analyses were applied to evaluate the risk stratification ability of MHR for hyperuricemia. In the ROC analysis, MHR as a single indicator had a statistically significant identification of hyperuricemia, but the AUC value was too low to be practically applied, and then we incorporated MHR into a clinical risk factors model and the results showed a significant improvement in risk identification of hyperuricemia $(0.718$ 
vs $0.724, p=0.008)$. To further confirm the capacity of MHR to optimize the risk stratification of hyperuricemia, we conducted reclassification analysis including both IDI and NRI which can assess the incremental potential of adding a new risk marker into an established risk model $[45,46]$. As expected, both results of category-free NRI and IDI presented a significant improvement in stratifying hyperuricemia risk when MHR was introduced into the established model of risk factors. In summary, our results suggest that MHR has the ability to optimize the risk stratification of hyperuricemia.

This study exists several limitations, which should be taken into account when considering the results. The first disadvantage is that cross-sectional design can only suggest the correlation between MHR and hyperuricemia, but the causality of this association needs to be confirmed by further prospective studies. Second, the study participants were enrolled from 26 rural areas in northeastern China, therefore, whether our results are applicable to the general Chinese population still deserves more studies to evaluate. Third, possible unmeasured confounding variables may exist and could have affected the results. The present study has adjusted age, sex, race, education level, family annual income, physical activity, current smoking, current drinking, BMI, eGFR, TC, HDL-C, SBP, FPG, lipid-lowering drug, and history of CVD, all of which are potential confounding, because they affect both exposure and outcome. However, there may be some variables that were not included in our analyses but can affect hyperuricemia, such as gout and hypouricemic drugs. Finally, considering the economic feasibility of epidemiological study, traditional inflammation markers such as CRP, ferritin and interleukin 6, which could be used to compare the predictive power with MHR for hyperuricemia, were not collected in present study. Therefore, whether MHR is a better predictor for hyperuricemia than traditional inflammation markers still needs further studies and evaluation.

\section{Conclusions}

In conclusion, MHR as a novel and simple marker of inflammation was independently associated with the prevalent hyperuricemia in a rural Chinese population. Our results also suggest the important value of MHR to optimize the risk stratification and prevention of hyperuricemia. However, our results should be verified in large prospective studies to explain the definite mechanism of MHR in hyperuricemia.

\footnotetext{
Abbreviations

MHR: Monocyte to high-density lipoprotein ratio; SUA: Serum uric acid; CNY: Chinese currency; CRP: C-reactive protein; CKD-EPI: Chronic Kidney Disease Epidemiology Collaboration; BMI: Body mass index; SBP: Systolic blood pressure; DBP: Diastolic blood pressure; Scr: Serum creatinine; eGFR: Estimated glomerular filtration rate; FPG: Fasting plasma glucose; TC: Total cholesterol; TG: Triglyceride; HDL-C: High-density lipoprotein
}

cholesterol; LDL-C: Low-density lipoprotein cholesterol; WBC: White blood cell; CVD: Cardiovascular disease; NCRCHS: Northeast China Rural Cardiovascular Health Study; OR: Odds ratio; EDTA: Ethylenediaminetetraacetic acid; Cl: Confidence interval; SD: Standard deviation; AUC: Area under the curve; Cl: Confidence interval; NRI: Net reclassification improvement; IDI: Integrated discrimination improvement

\section{Acknowledgments}

We would like to express our gratitude to all those who exert their effects in achieving this study.

\section{Authors' contributions}

In this study, MQ Chen and WR Shi did the study design, statistical analyses and results interpretation. CN Shi and YP Zhou participated as analyzing and resolving difficulties of analytic strategies and results discussion. Finally, YX Sun functioned as final reviewer and corresponding author. All authors read and approved the final manuscript.

\section{Funding}

This study was supported by grants from "Thirteenth Five-Year" program. funds (The National Key Research and Development Program of China, Grant. \#2017YFC1307600) and the National Key Research and Development Program from the Ministry of Science and Technology of China (Project Grant \#2018YFC1312400, Sub-project Grant \#2018YFC1312403).

\section{Availability of data and materials}

The datasets used and/or analyzed during the current study are available from the corresponding author on reasonable request.

\section{Ethics approval and consent to participate}

This study was conducted in compliance with the ethical principle of the Declaration of Helsinki. All participants provided written informed consent and all procedures were performed in accordance with the ethical standards. The study protocol was approved by the Ethics Committee of China Medical University (Shenyang, China).

\section{Consent for publication}

All co-authors and participants have given their consent for publication of this article in Lipids in Health and Disease.

\section{Competing interests}

The authors declare that they have no competing interests.

\section{Author details}

'Department of Cardiology, The First Hospital of China Medical University, 155 Nanjing North Street, Heping District, Shenyang 110001, China.

${ }^{2}$ Department of Neurology, The First Hospital of China Medical University, 155 Nanjing North Street, Heping District, Shenyang 110001, China.

Received: 31 October 2019 Accepted: 9 March 2020

Published online: 16 March 2020

\section{References}

1. Dalbeth N, Merriman TR, Stamp LK. Gout. Lancet. 2016;388:2039-52.

2. Tomiyama H, Shiina K, Vlachopoulos C, Iwasaki Y, Matsumoto C, Kimura K, Fujii M, Chikamori T, Yamashina A. Involvement of arterial stiffness and inflammation in Hyperuricemia-related development of hypertension. Hypertension. 2018;72:739-45.

3. Liu J, Tao L, Zhao Z, Mu Y, Zou D, Zhang J, Guo X. Two-year changes in Hyperuricemia and risk of diabetes: a five-year prospective cohort study. J Diabetes Res. 2018;2018:6905720.

4. Borghi C, Rosei EA, Bardin T, Dawson J, Dominiczak A, Kielstein JT, Manolis AJ, Perez-Ruiz F, Mancia G. Serum uric acid and the risk of cardiovascular and renal disease. J Hypertens. 2015;33:1729-41 discussion 1741.

5. Chiang KM, Tsay YC, Vincent Ng TC, Yang HC, Huang YT, Chen CH, Pan WH. Is Hyperuricemia, an early-onset metabolic disorder, causally associated with cardiovascular disease events in Han Chinese? J Clin Med. 2019;8:1202.

6. Johnson RJ, Bakris GL, Borghi C, Chonchol MB, Feldman D, Lanaspa MA, Merriman TR, Moe OW, Mount DB, Sanchez Lozada LG, et al. Hyperuricemia, acute and chronic kidney disease, hypertension, and cardiovascular disease: 
report of a scientific workshop organized by the National Kidney Foundation. Am J Kidney Dis. 2018;71:851-65.

7. Cachofeiro V, Goicochea M, de Vinuesa SG, Oubina P, Lahera V, Luno J. Oxidative stress and inflammation, a link between chronic kidney disease and cardiovascular disease. Kidney Int Suppl. 2008;74:S4-9.

8. Martinez BK, White CM. The emerging role of inflammation in cardiovascular disease. Ann Pharmacother. 2018;52:801-9.

9. Lu W, Xu Y, Shao X, Gao F, Li Y, Hu J, Zuo Z, Shao X, Zhou L, Zhao Y, Cen X. Uric acid produces an inflammatory response through activation of NFkappaB in the hypothalamus: implications for the pathogenesis of metabolic disorders. Sci Rep. 2015;5:12144.

10. Baldwin W, McRae S, Marek G, Wymer D, Pannu V, Baylis C, Johnson RJ, Sautin YY. Hyperuricemia as a mediator of the proinflammatory endocrine imbalance in the adipose tissue in a murine model of the metabolic syndrome. Diabetes. 2011;60:1258-69.

11. Spiga R, Marini MA, Mancuso E, Di Fatta C, Fuoco A, Perticone F, Andreozzi F, Mannino GC, Sesti G. Uric acid is associated with inflammatory biomarkers and induces inflammation via activating the NF-kappaB signaling pathway in HepG2 cells. Arterioscler Thromb Vasc Biol. 2017:37:1241-9.

12. Kundi H, Gok M, Kiziltunc E, Cetin M, Cicekcioglu H, Cetin ZG, Karayigit O, Ornek E. Relation between monocyte to high-density lipoprotein cholesterol ratio with presence and severity of isolated coronary artery Ectasia. Am J Cardiol. 2015;116:1685-9.

13. Acikgoz SK, Acikgoz E, Sensoy B, Topal S, Aydogdu S. Monocyte to highdensity lipoprotein cholesterol ratio is predictive of in-hospital and five-year mortality in ST-segment elevation myocardial infarction. Cardiol J. 2016;23: 505-12.

14. Murphy AJ, Woollard KJ, Hoang A, Mukhamedova N, Stirzaker RA, McCormick SP, Remaley AT, Sviridov D, Chin-Dusting J. High-density lipoprotein reduces the human monocyte inflammatory response. Arterioscler Thromb Vasc Biol. 2008;28:2071-7.

15. Mestas J, Ley K. Monocyte-endothelial cell interactions in the development of atherosclerosis. Trends Cardiovasc Med. 2008;18:228-32.

16. Woollard KJ, Geissmann F. Monocytes in atherosclerosis: subsets and functions. Nat Rev Cardiol. 2010;7:77-86.

17. Wei XB, Chen F, Huang JL, He PC, Wei YX, Tan N, Chen JY, Yu DQ, Liu YH. Novel risk biomarker for infective endocarditis patients with Normal left ventricular ejection fraction- monocyte to high-density lipoprotein cholesterol ratio. Circ J. 2017;82:283-8.

18. Thacker SG, Zarzour A, Chen Y, Alcicek MS, Freeman LA, Sviridov DO, Demosky SJ Jr, Remaley AT. High-density lipoprotein reduces inflammation from cholesterol crystals by inhibiting inflammasome activation. Immunology. 2016;149:306-19.

19. Yilmaz $M$, Kayancicek $H$. A new inflammatory marker: elevated monocyte to HDL cholesterol ratio associated with smoking. J Clin Med. 2018;7:76.

20. Canpolat U, Cetin EH, Cetin S, Aydin S, Akboga MK, Yayla C, Turak O, Aras D, Aydogdu S. Association of Monocyte-to-HDL cholesterol ratio with slow coronary flow is linked to systemic inflammation. Clin Appl Thromb Hemost. 2016:22:476-82

21. Uslu AU, Sekin Y, Tarhan G, Canakci N, Gunduz M, Karagulle M. Evaluation of monocyte to high-density lipoprotein cholesterol ratio in the presence and severity of metabolic syndrome. Clin Appl Thromb Hemost. 2018;24:828-33.

22. Chen JW, Li C, Liu ZH, Shen Y, Ding FH, Shu XY, Zhang RY, Shen WF, Lu L Wang XQ. The role of monocyte to high-density lipoprotein cholesterol ratio in prediction of carotid intima-media thickness in patients with type 2 diabetes. Front Endocrinol (Lausanne). 2019;10:191.

23. Satilmis $\mathrm{S}$. Role of the monocyte-to-high-density lipoprotein ratio in predicting atrial high-rate episodes detected by cardiac implantable electronic devices. North Clin Istanb. 2018;5:96-101.

24. Ya G, Qiu Z, Tianrong P. Relation of monocyte/high-density lipoprotein cholesterol ratio with coronary artery disease in type 2 diabetes mellitus. Clin Lab. 2018;64:901-6.

25. Li Z, Bai Y, Guo X, Zheng L, Sun Y, Roselle AM. Alcohol consumption and cardiovascular diseases in rural China. Int J Cardiol. 2016:215:257-62.

26. Shi WR, Wang HY, Chen S, Guo XF, Li Z, Sun YX. Estimate of prevalent diabetes from cardiometabolic index in general Chinese population: a community-based study. Lipids Health Dis. 2018;17:236

27. Wang HY, Shi WR, Yi X, Wang SZ, Luan SY, Sun YX. Value of reduced glomerular filtration rate assessment with cardiometabolic index: insights from a population-based Chinese cohort. BMC Nephrol. 2018;19:294.
28. Levey AS, Stevens LA, Schmid CH, Zhang YL, Castro AF 3rd, Feldman HI, Kusek JW, Eggers P, Van Lente F, Greene T, Coresh J. A new equation to estimate glomerular filtration rate. Ann Intern Med. 2009;150:604-12.

29. Kanbay M, Solak Y, Unal HU, Kurt YG, Gok M, Cetinkaya H, Karaman M, Oguz $Y$, Eyileten T, Vural A, et al. Monocyte count/HDL cholesterol ratio and cardiovascular events in patients with chronic kidney disease. Int Urol Nephrol. 2014;46:1619-25.

30. Gois PHF, Souza ERM. Pharmacotherapy for hyperuricemia in hypertensive patients. Cochrane Database Syst Rev. 2017:4:Cd008652.

31. Kundi H, Kiziltunc E, Cetin M, Cicekcioglu H, Cetin ZG, Cicek G, Ornek E. Association of monocyte/HDL-C ratio with SYNTAX scores in patients with stable coronary artery disease. Herz. 2016;41:523-9.

32. Ghattas A, Griffiths HR, Devitt A, Lip GY, Shantsila E. Monocytes in coronary artery disease and atherosclerosis: where are we now? J Am Coll Cardiol. 2013;62:1541-51.

33. Ammirati E, Moroni F, Magnoni M, Di Terlizzi S, Villa C, Sizzano F, Palini A, Garlaschelli K, Tripiciano F, Scotti I, et al. Circulating CD14+ and CD14(high)CD16- classical monocytes are reduced in patients with signs of plaque neovascularization in the carotid artery. Atherosclerosis. 2016;255: $171-8$.

34. Parthasarathy S, Barnett J, Fong LG. High-density lipoprotein inhibits the oxidative modification of low-density lipoprotein. Biochim Biophys Acta. 1990;1044:275-83

35. Yvan-Charvet $L$, Pagler T, Gautier EL, Avagyan S, Siry RL, Han S, Welch CL, Wang N, Randolph GJ, Snoeck HW, Tall AR. ATP-binding cassette transporters and HDL suppress hematopoietic stem cell proliferation. Science. 2010;328:1689-93.

36. Danesh J, Wheeler JG, Hirschfield GM, Eda S, Eiriksdottir G, Rumley A, Lowe GD, Pepys MB, Gudnason V. C-reactive protein and other circulating markers of inflammation in the prediction of coronary heart disease. N Engl J Med. 2004:350:1387-97.

37. Frohlich M, Imhof A, Berg G, Hutchinson WL, Pepys MB, Boeing H, Muche $R$ Brenner $\mathrm{H}$, Koenig W. Association between C-reactive protein and features of the metabolic syndrome: a population-based study. Diabetes Care. 2000; 23:1835-9.

38. Kang DH, Park SK, Lee IK, Johnson RJ. Uric acid-induced C-reactive protein expression: implication on cell proliferation and nitric oxide production of human vascular cells. J Am Soc Nephrol. 2005;16:3553-62.

39. Ficociello LH, Rosolowsky ET, Niewczas MA, Maselli NJ, Weinberg JM, Aschengrau A, Eckfeldt JH, Stanton RC, Galecki AT, Doria A, et al. Highnormal serum uric acid increases risk of early progressive renal function loss in type 1 diabetes: results of a 6-year follow-up. Diabetes Care. 2010;33: 1337-43.

40. Testa A, Mallamaci F, Spoto B, Pisano A, Sanguedolce MC, Tripepi G, Leonardis D, Zoccali C. Association of a polymorphism in a gene encoding a urate transporter with CKD progression. Clin J Am Soc Nephrol. 2014;9: 1059-65.

41. Tseng $W C$, Chen $Y T$, Lin YP, Ou SM, Yang CY, Lin CH, Tarng DC. Hyperuricemia predicts an early decline in renal function among older people: a community-based cohort study. Sci Rep. 2019;9:980.

42. Sato Y, Feig DI, Stack AG, Kang DH, Lanaspa MA, Ejaz AA, Sanchez-Lozada $L G$, Kuwabara M, Borghi C, Johnson RJ. The case for uric acid-lowering treatment in patients with hyperuricaemia and CKD. Nat Rev Nephrol. 2019; 15(12):767-75.

43. Shi WR, Wang HY, Chen S, Guo XF, Li Z, Sun YX. The impact of monocyte to high-density lipoprotein ratio on reduced renal function: insights from a large population. Biomark Med. 2019;13(9):773-83.

44. Hughes K, Flynn T, de Zoysa J, Dalbeth N, Merriman TR. Mendelian randomization analysis associates increased serum urate, due to genetic variation in uric acid transporters, with improved renal function. Kidney Int. 2014:85:344-51.

45. Pencina MJ, D'Agostino RB Sr, D'Agostino RB Jr, Vasan RS. Evaluating the added predictive ability of a new marker: from area under the ROC curve to reclassification and beyond. Stat Med. 2008;27:157-72 discussion 207-112.

46. Pencina MJ, D'Agostino RB Sr, Steyerberg EW. Extensions of net reclassification improvement calculations to measure usefulness of new biomarkers. Stat Med. 2011;30:11-21.

\section{Publisher's Note}

Springer Nature remains neutral with regard to jurisdictional claims in published maps and institutional affiliations. 